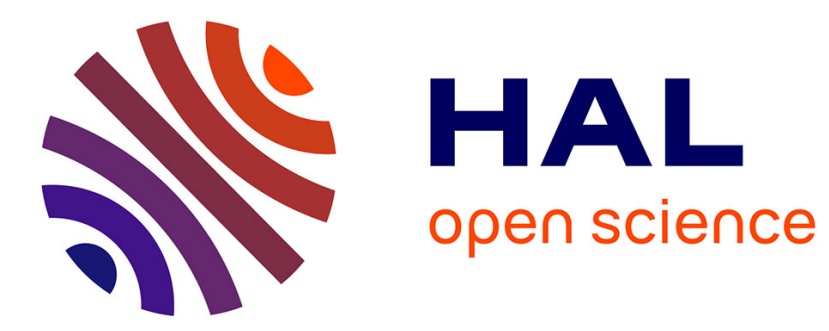

\title{
A millimeter-wave Bessel beam launcher through the excitation of higher-order leaky modes
}

Walter Fuscaldo, Guido Valerio, A. Galli, Ronan Sauleau, Mauro Ettorre

\section{To cite this version:}

Walter Fuscaldo, Guido Valerio, A. Galli, Ronan Sauleau, Mauro Ettorre. A millimeter-wave Bessel beam launcher through the excitation of higher-order leaky modes. EuCAP 2014, 8th European Conference on Antennas and Propagation, Apr 2014, The Hague, Netherlands. pp.1439-1441, 10.1109/EuCAP.2014.6902051 . hal-01114110

\author{
HAL Id: hal-01114110 \\ https://hal.science/hal-01114110
}

Submitted on 19 Feb 2016

HAL is a multi-disciplinary open access archive for the deposit and dissemination of scientific research documents, whether they are published or not. The documents may come from teaching and research institutions in France or abroad, or from public or private research centers.
L'archive ouverte pluridisciplinaire HAL, est destinée au dépôt et à la diffusion de documents scientifiques de niveau recherche, publiés ou non, émanant des établissements d'enseignement et de recherche français ou étrangers, des laboratoires publics ou privés. 


\title{
A Millimeter-Wave Bessel Beam Launcher through the Excitation of Higher-Order Leaky Modes
}

\author{
Walter Fuscaldo ${ }^{1,2}$, Guido Valerio ${ }^{1}$, Alessandro Galli ${ }^{2}$, Ronan Sauleau ${ }^{1}$, and Mauro Ettorre ${ }^{1}$ \\ ${ }^{1}$ Institut d'Electronique et de Télécommunications de Rennes (IETR), UMR CNRS 6164, \\ Université de Rennes 1, 35042 Rennes, France \\ ${ }^{2}$ Department of Information Engineering, Electronics and Telecommunications (DIET), \\ Sapienza University of Rome, 00184 Rome, Italy
}

\begin{abstract}
We present a Bessel beam launcher based on a radial leaky-wave antenna operating at millimeter waves. With respect to a design method proposed by one of the Authors in a lower frequency range, a new approach is chosen here, as an alternative to a simple scaling of all the geometrical parameters. In order to avoid inacceptable levels of losses and possible fabrication issues due to an extremely thin substrate, our design is based on the suitable excitation of a higher-order leaky mode. Closed-form equations are derived in order to simplify the design, providing reliable values for the physical parameters once the near-field characteristics are assigned. Simulated results are shown to validate the proposed procedure.
\end{abstract}

Index Terms-Bessel beams, near-field focusing, artificial surface, leaky-wave modes, high-order modes, millimeter waves.

\section{INTRODUCTION}

Bessel beams are a class of solutions of Maxwell's equations which exhibit invariance of the transverse beam cross section along the direction of propagation [1]. Such features are appealing for several applications in the millimeter-wave range such as near-field communications, non-invasive imaging, or radiometry.

The development of Bessel beam launchers has been an argument of interest in optics, where several solutions have been proposed during the years. An ideal Bessel beam has an infinite transverse cross section described by $n^{\text {th }}$ order Bessel function $J_{n}(\cdot)$, thus leading to an infinite energy. Realizable beams require then a lateral truncation, limiting the so-called non-diffractive range, i.e., the distance from the screen defining a region of space where the beam propagates with invariant cross section. In the spectral domain, a Bessel beam is described by a single ring (annulus), selected by the radial field variation of the Bessel function. A scalar theory is often sufficient at optical frequencies to describe such beams. This assumption is strictly valid if the size of the aperture is much larger than the non-diffractive distance, and if the beam cross section is large in terms of wavelengths.

The authors would like to thank the European Science Foundation (NEWFOCUS project), and Rennes Métropole (AIS project). This work was performed using HPC resources from GENCI-IDRIS (grant 2013050779).
Unfortunately, such an extensive analysis in the optical range does not have correspondence in the microwave and millimeter-wave range. In these cases, the vector nature of the beam cannot be neglected due to the different electrical dimensions of the device. A solution to the problem is proposed in [2] and [3] through a low-profile and low-cost prototype working at $10 \mathrm{GHz}$, based on a leaky radial waveguide (see Fig. 1). A grounded slab is covered by an artificial surface supporting a leaky-wave mode with the same spectral content of the required Bessel beam. The waveguide was shorted at its end with a vertical metallic rim, to reflect the incident cylindrical wave and to shape the field on the aperture according to the required Bessel-function law. A central coaxial cable excites the lowest-order TM leaky wave, whose radial wavenumber is tuned by correctly choosing the thickness of the substrate and the equivalent impedance of the artificial surface. Bessel beams of arbitrary transverse size can be synthesized through this approach, thanks to the rigorous derivation of the design rules from the complete vector form of Maxwell's equations.

In this work, we aim at the design of a Bessel-beam launcher working around $40 \mathrm{GHz}$. A simple scaling of the structure shown in [3] would lead to a very thin structure, posing problems due to an unbearable level of ohmic losses.

In order to limit losses, the thickness of the grounded slab is kept larger and a higher-order leaky-wave mode is excited instead.

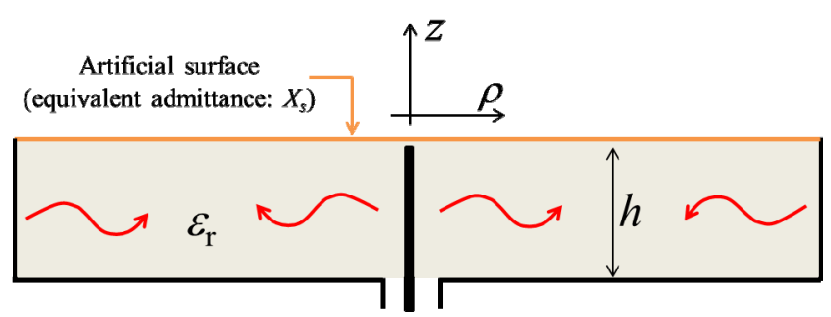

Fig. 1. Structure under analysis as a Bessel beam launcher, with physical and geometrical parameters (section view). The red arrows show the incident and reflected cylindrical waves launched by a central coaxial-cable probe. 
New closed-form equations are obtained for the proper choice of the geometrical and physical parameters, once the Bessel-beam required features are selected.

In Section II, a dispersive analysis is performed on the structure shown in Fig. 1, to characterize the various leaky modes contributing to the aperture fields in different frequency ranges. In Section III the design of the structure is described, and in Section IV the synthesis of the artificial surface and the simulations of the final model are presented.

\section{DisPERSIVE ANALYSIS OF THE STRUCTURE}

The performance of a Bessel beam launcher is strongly affected by the propagation and radiation characteristics of the leaky wave excited [2]. A rigorous dispersive analysis of the stratification is then required in order to select the best geometrical and physical parameters leading to the required leaky wavenumber.

In order to investigate the possible leaky modes supported by our structure in a wide range of frequencies, we solve the transverse resonant equation of the relevant layered structure [4], defining the frequency dependence of the complex radial wavenumbers $k_{\rho}=\beta-j \alpha$ of the TM modes

$$
\frac{\omega \varepsilon_{0}}{k_{z 0}}+Y_{\mathrm{s}}-j \frac{\omega \varepsilon_{0} \varepsilon_{\mathrm{r}}}{k_{z}} \cot \left(k_{z} h\right)=0,
$$

where $Y_{s}$ is the artificial-surface equivalent admittance, $k_{z 0}=\sqrt{k_{0}^{2}-k_{\rho}^{2}}$ and $k_{z}=\sqrt{k_{0}^{2} \varepsilon_{\mathrm{r}}-k_{\rho}^{2}}, k_{0}$ being the freespace wavenumber and $\varepsilon_{\mathrm{r}}$ the dielectric constant of the slab. For simplicity, the presence of the glue is neglected here. The square root determination is chosen in such a way that $\operatorname{Im}\left\{k_{z}\right\}>0$, since we are looking for forward leaky-wave mode, characterized by an improper behavior along $z$ direction (i.e., an exponential rise) [4].

Once the thickness of the substrate and the impedance of the artificial surface have been fixed (the specific parameters are given in the caption of Fig. 2), we can easily perform an analysis over a very wide frequency range. The results of such analysis are shown in Fig. 2 by plotting the phase and attenuation constants normalized with respect to $k_{0}$.

At low frequencies [Fig. 2(a)], we encounter the lowestorder leaky wave $\mathrm{TM}_{0}$, used in [2]. As seen in Fig. 2, a low frequency region is found, dominated by the attenuation constant $\alpha$ over the phase constant $\beta$. On the other hand, at higher frequencies, $\beta>\alpha$. In order to distinguish the two behaviors, we can define a cutoff frequency $f_{\mathrm{c} 0}$ by substituting the approximation $k_{\rho} \cong 0$ in (1). Since small values of $\alpha$ are always required in order to illuminate the aperture with a Bessel profile [2], the mode $\mathrm{TM}_{0}$ can be used in a Bessel beam launcher only at frequencies $f>f_{\mathrm{c} 0}$.

As frequency increases, the $\mathrm{TM}_{0}$ mode becomes a bound surface wave (i.e., $\beta>k_{0}$ ), and is no longer suitable for our application. For this reason, in Fig. 2(b), around the central frequency $33 \mathrm{GHz}$, we show only the new $\mathrm{TM}_{1}$ leaky wave, becoming relevant to our analysis.

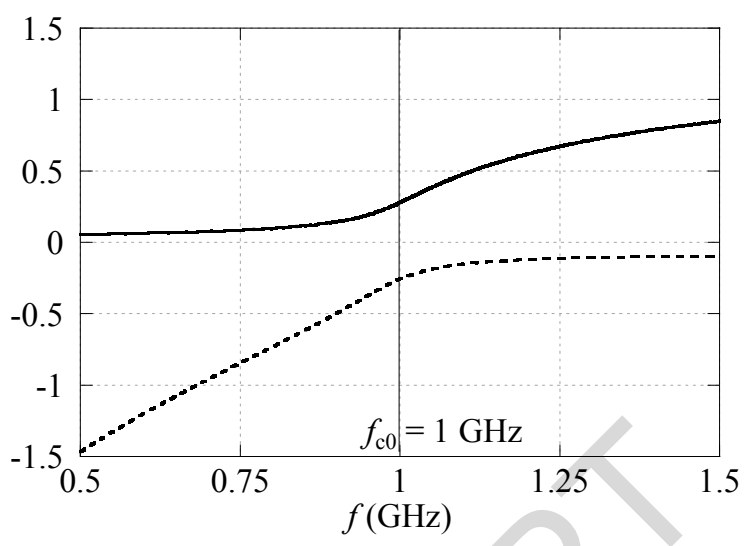

(a)

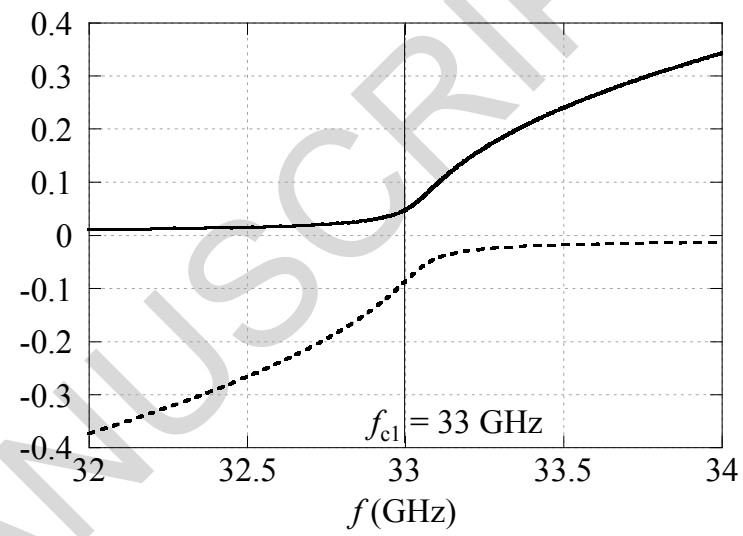

(b)

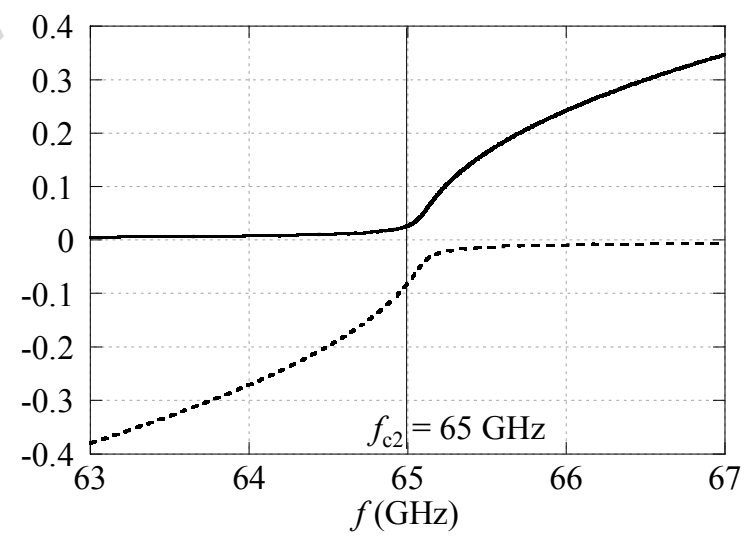

(c)

Fig. 2. Normalized phase $\beta / k_{0}$ (solid lines) and attenuation $\alpha / k_{0}$ (dashed lines) constants of TM leaky-wave modes in different frequency ranges. (a) Frequency range $[0.5,1.5] \mathrm{GHz}$, constants and cutoff frequency $f_{\mathrm{c} 0}$ of the $\mathrm{TM}_{0}$ leaky mode. (b) Frequency range $[32,34] \mathrm{GHz}$, constants and cutoff frequency $f_{\mathrm{cl}}$ of the $\mathrm{TM}_{1}$ leaky mode. (c) Frequency range $[63,67] \mathrm{GHz}$, constants and cutoff frequency $f_{\mathrm{c} 2}$ of the $\mathrm{TM}_{2}$ leaky mode. Geometrical and physical parameters: $\varepsilon_{\mathrm{r}}=2.17$, $h=3.175 \mathrm{~mm}, Y_{\mathrm{s}}=\mathrm{j} 40 \mathrm{mS}$.

Also in this case, we can define a cutoff frequency $f_{\mathrm{cl}}$, and the qualitative behavior of this new mode is the same as the one discussed for the $\mathrm{TM}_{0}$. For this reason, this $\mathrm{TM}_{1}$ 
mode is a good candidate for the excitation of a Bessel beam at frequencies higher than $f_{\mathrm{c}}$, where the previous $\mathrm{TM}_{0}$ mode is a surface wave and cannot excite a Bessel beam.

A similar evolution of the leaky wavenumbers can be observed at even higher frequencies in Fig. 2(c), where also the $\mathrm{TM}_{1}$ mode becomes a surface wave. Again, a third-order leaky mode $\mathrm{TM}_{2}$ is found having cutoff at $f_{\mathrm{c} 2}=65 \mathrm{GHz}$. Such a mode could be useful to excite a Bessel profile on the aperture if the same structure were used at frequencies higher than $f_{\mathrm{c} 2}$.

Since the Bessel launcher proposed in this paper is designed to work around $40 \mathrm{GHz}$, we retain the structure described in the caption of Fig. 2, having similar parameters as in [2], and we will consider the excitation of the $\mathrm{TM}_{1}$ leaky-wave mode.

\section{DESIGN OF THE STRUCTURE}

In order to gain a physical insight into the propagation mechanisms of the waves supported by the structure in Fig. 1, we try to obtain simple closed-form expressions relating the leaky wavenumber and the parameters of interest.

Such a result was already obtained in [2], where the assumption of thin layer $k_{z} h<<1$ is used in (1) in order to approximate the cotangent as $\cot \left(k_{z} h\right) \cong 1 /\left(k_{z} h\right)$. This simplified hypothesis no longer holds in this context, where the vertical field variation of the higher-order modes is not negligible. On the other hand, if we choose a thickness $h$ close to an integer multiple $m$ of half-wavelengths, the cotangent function can be approximated through a firstorder Laurent series.

This approximation leads to the equation

$$
\frac{\omega \varepsilon_{0}}{k_{z 0}}+Y_{\mathrm{s}}-j \frac{\omega \varepsilon_{0} \varepsilon_{\mathrm{r}}}{k_{z}} \frac{1}{k_{z} h+m \pi}=0,
$$

which allows us to derive a closed-form result for the admittance $Y_{s}$ as a function of the requested wavenumber $k_{\rho}$ and the thickness $h$. The final formulas are not reported here for the sake of brevity.

\section{NUMERICAL RESULTS}

\section{A. Validation of the Bessel Beam Launcher}

A Bessel beam having transverse propagation constant $k_{\rho}=(0.8-j 0.007) k_{0}$ has specifically been designed. The structure obtained from the transverse resonant method has been simulated with COMSOL Multiphysics, to verify the possibility to excite a Bessel beam propagating within the expected non-diffractive range. The artificial surface is here modeled as an infinitesimally-thin layer, regardless of its actual implementation. In Fig. 3, the transverse profile of the normalized vertical electric field at different distances from the waveguide aperture is shown. The typical Bessel function $J_{0}$ profile is observed up to the non-diffractive range, equal to $16.7 \mathrm{~mm} \cong 2.23 \lambda$, where the sidelobes are no longer clearly distinct and the main beam starts to widen.

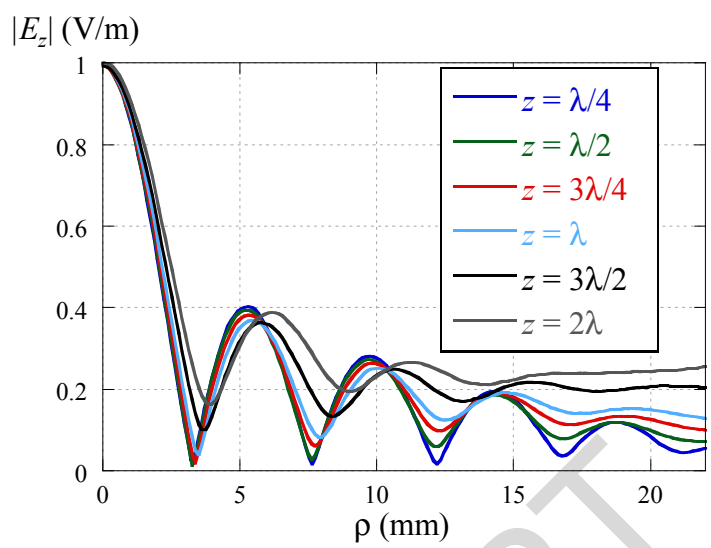

Fig. 3. Transverse profile of the magnitude of the vertical electric field $\left|E_{z}\right|$ at different distances from the aperture, from COMSOL Multiphysics. Each curve is normalized to its maximum value.

\section{B. Design of the Complete Structure}

The artificial surface is synthesized through a layer of permittivity $\varepsilon_{\mathrm{r} \text {,glue }}=6.15$ (duroid 6006). On its two interfaces, two-dimensional arrays of interleaved squared metallic patches are printed [3]. The equivalent admittance is extracted from the simulation of the reflection coefficient due to a normally-incident plane wave, through the commercial finite-element solver HFSS. The thickness of the layer and the period between patches are sufficiently small to justify the modelling as an equivalent surface.

Once the surface is designed, it is included in an HFSS model with all the realistic details of the design: this can take into account, e.g., the presence of the glue and of a matching network specifically designed in order to optimize the input matching of the final prototype.

\section{CONCLUSION}

We have presented the design of a radial waveguide operating at around $40 \mathrm{GHz}$ on a higher-order leaky mode for Bessel beam excitation. The use of a higher-order mode leads to a thicker substrate, capable to reduce losses at millimeter waves. Exhaustive details on the dispersive properties of the selected structures have been discussed in different frequency ranges, showing the dispersive behaviors of TM leaky-wave modes in connection with their use in Bessel beam launchers.

\section{REFERENCES}

[1] J. Durnin, "Exact solutions for nondiffracting beams. I. The scalar theory," J. Opt. Soc. Amer. A., vol. 4, no. 4, pp. 651-654, Apr. 1987.

[2] M. Ettorre and A. Grbic, "Generation of propagating Bessel beams using leaky-wave modes," IEEE Trans. Antennas Propag., vol. 60, no. 8, pp. 3605-3613, Aug. 2012.

[3] M. Ettorre, S. M. Rudolph, and A. Grbic, "Generation of propagating Bessel beams using leaky-wave modes: experimental validation," IEEE Trans. Antennas Propag., vol. 60, no. 7, pp. 2645-2653, Jul. 2012.

[4] G. Valerio, D. R. Jackson, and A. Galli, "Fundamental properties of surface waves in lossless stratified structures," Proc. R. Soc. A, vol. 466, pp. 2447-2469, 2010. 